\title{
Excitation energy and pair correlation function of trions near an LiF surface
}

\author{
Beate Solleder, ${ }^{1}$ Ludger Wirtz, ${ }^{2}$ and Joachim Burgdörfer ${ }^{1}$ \\ ${ }^{1}$ Institute for Theoretical Physics, Vienna University of Technology, 1040 Vienna, Austria, EU \\ ${ }^{2}$ Institute for Electronics, Microelectronics, and Nanotechnology (CNRS UMR 8520), Department ISEN, \\ 59652 Villeneuve d'Ascq Cedex, France, EU \\ (Received 3 June 2008; revised manuscript received 19 September 2008; published 28 October 2008)
}

\begin{abstract}
We investigate the excitation spectrum of a singly charged LiF surface using an embedded cluster approach. The spectrum is determined by multiconfiguration self-consistent field calculations. We find stable trionic states, i.e., quasimolecular states of a three-quasiparticle complex consisting of two holes and one electron interacting through the Coulomb potential. The excitation energy of the lowest trionic state is approximately $12.1 \mathrm{eV}$. The mean hole-hole spacing is about 7.6 a.u. (or $4 \AA$ ). Our results confirm the interpretation of experiments [P. Roncin et al., Phys. Rev. Lett. 89, 043201 (2002)] where trion excitation was suggested as effective energy-loss channel during the neutralization of $\mathrm{Ne}^{+}$ions at an $\mathrm{LiF}$ surface.
\end{abstract}

DOI: $10.1103 /$ PhysRevB.78.155432

PACS number(s): 71.35.Pq

\section{INTRODUCTION}

The interaction of charged particles with surfaces is routinely used to induce electronic excitations and thus to probe the excitation spectrum of surfaces. Of particular interest are wide-band gap insulators such as alkali halides where the threshold for inelastic processes is high $(\approx 10 \mathrm{eV})$ and accessible excitation channels in the bulk band gap due to surface effects are of particular importance for the stopping power and energy-loss spectra. For positively charged ions an additional reaction channel becomes available, charge transfer, which, in turn, can lead to potential sputtering of target atoms. In view of technical applications such as controlled nanoscale surface modifications or selective removal of insulating layers, a detailed understanding of these types of electronic excitations is essential.

Charge transfer accompanied by excitation of the surface may lead, in addition to single-hole creation, to the formation of excitation complexes involving more than two quasiparticles, such as an electron-bihole complex. Such a trion consists of one electron excited from the valence band bound to two holes (Fig. 1). The existence of a trion and other similar excitonic states was suggested by Lampert ${ }^{1}$ in 1958. The subsequent studies of trions during the next decades were focused, both experimentally and theoretically, on semiconductors. $^{2-10}$ To our knowledge, the first experimental evidence for a trionic state in $\mathrm{LiF}$ was found by Khemliche et $a l .{ }^{11}$ who studied the neutralization of $\mathrm{Ne}^{+}$ions during grazing incidence on a lithium fluoride surface. By measurement of the energy loss and final charge state of the projectile in coincidence with the energy spectrum of emitted electrons they identified two main neutralization channels. The first one is Auger neutralization where one F $2 p$ electron is captured from the surface into a low lying projectile state, while a second F $2 p$ electron is emitted. The second process differs from the first in that no additional electron is emitted. As resonant electron transfer can be ruled out due to a mismatch of the electronic levels involved, the detection of the neutral $\mathrm{Ne}^{0}$ final states unaccompanied by electron emission was explained by an Auger-type capture and excitation process where the transferred energy does not suffice to excite the

valence electron above the vacuum level. Instead, a hole and an electron-hole pair are created forming a trion, which constitutes a bound state within the band gap of LiF. From the measured projectile energy loss the trion excitation energy relative to the ground state of the singly charged surface was estimated to about $12.6 \mathrm{eV}$ and the mean binding energy relative to the ground state of the doubly charged system to $3.5 \pm 1 \mathrm{eV}$ which was confirmed in a later experiment using $\mathrm{F}^{+}$projectiles. $^{12}$

Earlier theoretical studies of trions in semiconductors were based on a three-body Hamiltonian for two holes and one electron using effective masses for the particles and dielectric constants. ${ }^{2-7,13}$ The corresponding Schrödinger equation was either solved numerically ${ }^{2-5}$ or simplified by restricting configuration space to lower dimensions ${ }^{13}$ or by fixing positions of the holes, ${ }^{6,7}$ thereby imposing a BornOppenheimer-like approximation. Three-body bound states in LiF was studied by Shibata et al. ${ }^{14}$ applying the Faddeev equation; however, only within a one-dimensional model where only one lithium and two adjacent fluorine ions are considered. The two holes are fixed to the fluorine sites and the electron to the lithium site. To our knowledge, $a b$ initio calculations for trions in $\mathrm{LiF}$ are not yet available. So far only excitonic excitations in the neutral system have been

a)

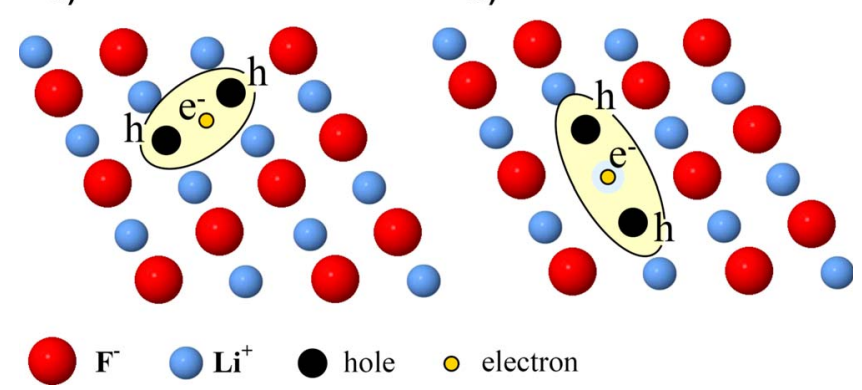

FIG. 1. (Color online) Formation of a trion on an $\mathrm{LiF}(001)$ surface, schematically. Two possible trion configurations are shown: (a) the two holes occupy nearest-neighbor fluorine sites (hole distance $\sqrt{2} a$ ) and (b) the holes are located at next-to-nearest-neighbor $\mathrm{F}^{-}$sites (hole distance $2 a$ ). 

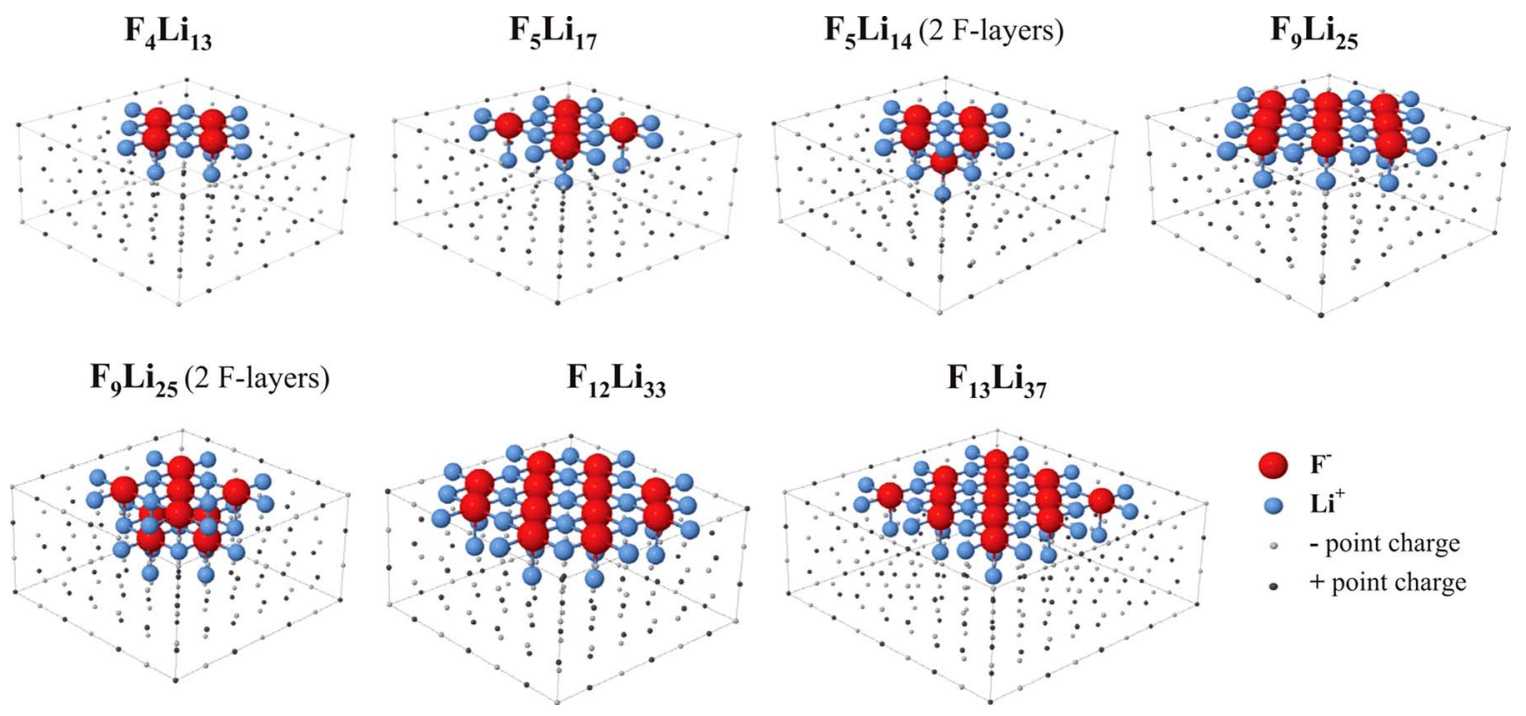

FIG. 2. (Color online) Representation of the LiF surface by embedded clusters of increasing size. Clusters studied in the present work are depicted.

treated on an ab initio level. The excitation energy of an exciton corresponds to the energy of an electron that has been excited to the conduction band bound by the attractive electron-hole interaction. The latter is taken into account by the Bethe-Salpeter equation (BSE). ${ }^{15}$ With this method, Wang et al. ${ }^{16}$ obtained for LiF a bulk exciton at $12.7 \mathrm{eV}$ and a surface exciton at $9.2 \mathrm{eV}$ (Refs. 17 and 18) above the ground-state energy.

In the present work we perform $a b$ initio calculations for trionic excitations in the singly charged system. We calculate the excitations in a finite-size $\mathrm{F}_{m}^{-} \mathrm{Li}_{n}^{+}$cluster embedded into a matrix of point charges that represent the remainder of the infinitely extended surface and bulk. For such a finite system we employ a quantum-chemistry approach. In our calculations the Schrödinger equation for all electrons (up to 204) of all atoms in the cluster is solved self-consistently. Excitation energies are calculated as the total-energy difference between ground and excited states. As Hartree-Fock (HF) calculations neglect correlations while trions represent strongly correlated particle-hole complexes, it is important to go beyond the HF level. We use the multiconfiguration self-consistent field (MCSCF) method. The configurations containing the electron-hole pairs that contribute dominantly to the trionic states are thereby included.

We systematically study clusters of increasing size up to 13 fluorine and 37 lithium ions to assess possible finite-size errors.

In Sec. II we briefly review the methods underlying our calculation. In Sec. III we present the excitation energies, binding energies, and the pair-correlation function characterizing the trion complex. The relation to the fundamental three-body Coulomb complexes in vacuum and simplified models for the solid-state environment will be analyzed in Sec. IV.

\section{THEORETICAL METHOD}

Starting point of our analysis of the electron-bihole complex is the multiconfiguration self-consistent field method. ${ }^{19}$
On the MCSCF level, the $N$-electron solution $\Psi\left(\overrightarrow{\mathbf{r}}_{1}, \ldots, \overrightarrow{\mathbf{r}}_{N}\right)$ of the stationary Schrödinger equation, with $\overrightarrow{\mathbf{r}}_{i}$ including all spatial and spin coordinates, is expressed as a linear combination of different configuration state functions (CSFs) $\Phi_{l}$,

$$
\Psi=\sum_{l} \alpha_{l} \Phi_{l}
$$

Every $\Phi_{l}$ is an $N$-electron Slater determinant corresponding to a certain distribution of the $N$ electrons over the molecular orbitals (MOs). These orbitals are expanded in the Gaussian basis set of Schäfer et $a l .{ }^{20}$ For the fluorine three $s$-type and two $p$-type Gaussians are used, while lithium orbitals are expanded in two $s$-type functions. The choice of this basis constitutes a compromise between accuracy and computational feasibility, the limit of which is rapidly reached with increasing number of active ions in the cluster. For a small cluster, we checked that increasing the basis size changes the resulting trion excitation energies by less than $0.5 \mathrm{eV}$. Through the superposition of many configuration state functions, correlation effects are taken into account in the MCSCF method as opposed to HF calculations where only one Slater determinant is considered. ${ }^{21}$ For the simultaneous calculation of the ground state and several excited states, a state averaged MCSCF is performed, i.e., the CSF expansion coefficients $\alpha_{l}$ are optimized separately for each state, while the MOs are determined such that the total energy averaged over all states is minimized.

We investigate seven different clusters (Fig. 2) where the total systems including the ions and all point charges consist of $7 \times 7 \times 4$ lattice sites for clusters with four to 12 fluorine ions while the $\mathrm{F}_{13} \mathrm{Li}_{37}$ cluster is embedded in a $9 \times 9 \times 4$ lattice. Every $\mathrm{F}^{-}$is surrounded by $\mathrm{Li}^{+}$ions to avoid artificial distortion of the electron density ${ }^{22}$ and all sites not occupied by ions are filled with point charges ensuring the proper inclusion of the Madelung potential. ${ }^{23}$ This embedded cluster approach has been successfully used in studies on ionic crystals (see Ref. 23 and references therein). 
In our calculations the positions of all nuclei are fixed. Effects of lattice distortions due to the excitation of a trion are neglected in the present study. As self-trapped excitons in alkali-halide crystals lead to relaxation of the lattice near the exciton, ${ }^{24}$ we would expect that the formation of trions also influences the lattice structure. The lattice relaxation around the holes may possibly lead to a stronger binding energy and a longer lifetime of the trion. However, due to the computational difficulty of geometry optimization for large clusters in an excited state, the study of self-trapping is beyond the scope of the present calculation.

In an MCSCF calculation, the different CSFs are generated by exciting one or several electron-hole pairs, i.e., one or several electrons in excited orbitals outside the groundstate configuration. For reasons of computational limitation we only allow excitations of valence electrons (from F $2 p$-like MOs) to the energetically lowest unoccupied orbitals (Li $2 s$-like MOs). Using the quantum chemistry code COLUMBUS, ${ }^{25}$ most of the MCSCF calculations are performed in the $A_{1}$ symmetry of the $C_{2 v}$ symmetry group which has the highest symmetry and thus should contain the lowest-lying excitation energy. Within this symmetry, a wavefunction remains unchanged under the symmetry operations, rotation by $180^{\circ}$, reflection at the $x-z$ plane and reflection at the $y-z$ plane, where $z$ denotes the direction of the surface normal. For consistency checks, additional calculations in the $B_{1}, B_{2}$, and $A_{2}$ symmetries were performed. We restrict the active space, i.e., the active orbitals to and from which excitations are allowed further by including only F $2 p$-like MOs of $A_{1}$ symmetry and the three lowest $A_{1}$ Li $2 s$-like MOs. Although these constraints may seem rather restrictive, up to $113 \mathrm{CFSs}$ of the most probable configurations are taken into account. A comparison with excitation energies calculated using a larger active space, which can be handled for small clusters, shows that the results do not change significantly. We observe differences in the $500 \mathrm{meV}$ range which is well within the overall accuracy level ( $\approx 1 \mathrm{eV}$ ) we aim for.

Representing the band structure by a finite cluster leads to a discretization of the band (Fig. 3), the density of which increases with increasing cluster size. The continuous band structure can be recovered by assigning each discrete level a finite (typically) Gaussian width. Also trionic excitations near the surface should feature a narrow band signifying the dispersion relation for propagation in the surface plane. The present calculation for finite clusters reproduces the energetically lowest trion state corresponding to the $k=0(\Gamma)$ point of the band which determines the threshold for the energy-loss channel. The determination of the trionic state is schematically illustrated in Fig. 4. Within the singly charged sector $(q=1)$, excited states are determined from configurations that contain already one hole. The lowest total energy (ground state) results from configurations with the (first) hole at the top of the valence band [Fig. 4(b)] corresponding to the hole with $k=0$ ( $\Gamma$ point), while excited states correspond to a distribution of the hole in lower-lying valence-band states (holes with finite $k$ ). The trionic state corresponds to the lowest particle-hole excitation starting from this $(q=1)$ onehole ground state. Note that the ordering in the total-energy diagram [Fig. 4(a)] is inverted relative to the single-particle (hole) energy-level diagram [Fig. 4(b)].

\section{cluster}

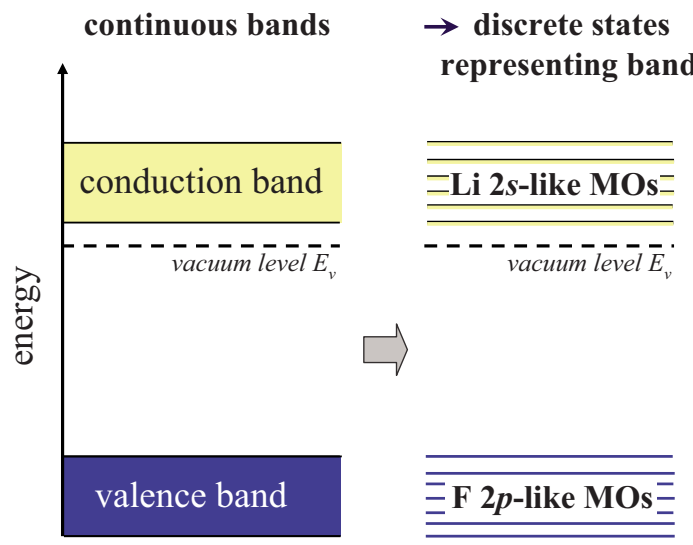

FIG. 3. (Color online) Continuous energy bands of LiF represented by discrete levels of the F $2 p$-like molecular orbitals (valence band) and the Li $2 s$-like orbitals (conduction band), schematically.

As mentioned above, there will be additional excited trion states of higher energy or, in the limit of an infinitely extended crystal, a dispersive band of trion states. We limit our studies to the energetically lowest trion state as the calculations of the first excited state are already computationally very demanding.

\section{TRION ENERGIES AND PAIR CORRELATION FUNCTIONS}

\section{A. Trion energy}

We present in the following excitation energies, binding energies, and wavefunctions for trions which are excited states in the band gap of the surface with asymptotic charge state $q=1$. It is instructive to compare these excitations to a) total energy diagram

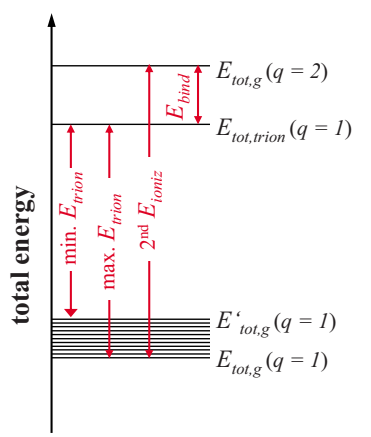

b) energy level diagram charged cluster $(q=1)$

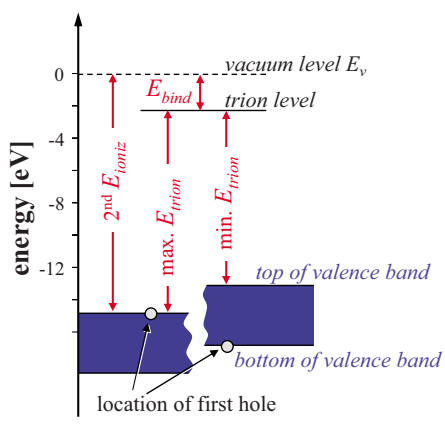

FIG. 4. (Color online) (a) Energy diagram of the total energies determined from MCSCF calculations. $E_{\text {tot, } g}(q=1)$ and $E_{\text {tot, }, g}^{\prime}(q=1)$ are the lowest and highest "ground states" of the singly charged cluster, respectively. $E_{\text {tot,trion }}(q=1)$ is the trion state and $E_{\text {tot, }, g}(q$ $=2$ ) is the ground state of the doubly charged cluster. (b) Singleparticle energy level diagram. Relative energies used in Table I are also shown. 


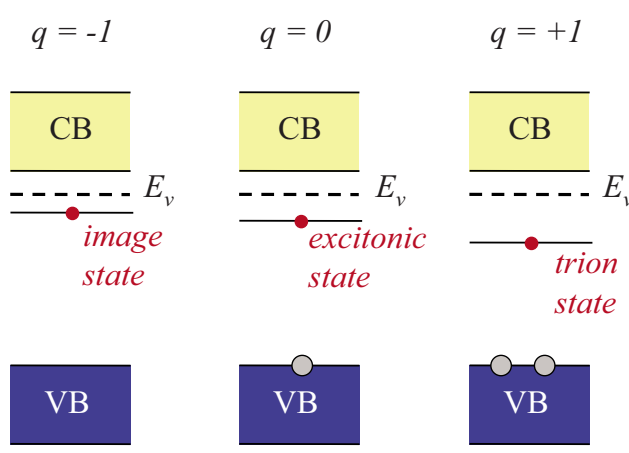

FIG. 5. (Color online) Surface excitations in the band gap of LiF for different charge states $q$ of the cluster, schematically (VB: valence band; $\mathrm{CB}$ : conduction band).

those in other charge sectors (Fig. 5) for which ab initio calculations are available.

Excitonic states in the neutral sector $q=0$ are well investigated, both experimentally and theoretically. In electronenergy-loss spectroscopy (EELS), thresholds for the excitation energy $E_{\text {exc }}$ relative to the neutral ground state were found at $E_{\mathrm{exc}}=9.7 \mathrm{eV}$ (Ref. 26) and approximately $9 \mathrm{eV}$ (Ref. 27). From other EELS studies, dominant excitation energies of $10.4 \mathrm{eV}$ (Ref. 28) and $10.3 \mathrm{eV}$ (Ref. 29) were obtained (threshold energies were not published in these cases), in agreement with Refs. 26 and 27 where the loss peaks were found at 10.65 and $10.2 \mathrm{eV}$, respectively. From energy-loss spectroscopy of low-energy protons ${ }^{30}$ an excitation energy of about $10.15 \mathrm{eV}$ can be derived with a threshold at about $9 \mathrm{eV}$ while electron-stimulated desorption (ESD) experiments yield a threshold for the excitation of surface excitons ${ }^{31}$ of $9.1 \pm 0.6 \mathrm{eV}$. For excitations in the neutral sector also accurate theoretical calculations are available. Numerical solutions of the Bethe-Salpeter equation yield for bulk excitons ${ }^{16}$ $E_{\text {exc }}=12.7 \mathrm{eV}$, for excitons near the surface with the electronic wavefunction confined to the surface layer ${ }^{16} E_{\text {exc }}$ $=12.3 \mathrm{eV}$. When the wavefunction is allowed to extend several angstroms into the vacuum by adding Gaussian orbitals centered above the surface ${ }^{17}$ the exciton energy is reduced to $E_{\text {exc }} \approx 9.2 \mathrm{eV}$.

We have calculated the energy of neutral excitons for clusters of different sizes to compare it with these data. This allows us to determine an upper bound for the systematic error of our method, which is better suited for the $q=1$ than for the $q=0$ sector. Unlike excitons, i.e., an electron-hole pair configuration with the hole localized near an F in the surface layer and the electronic cloud reaching out into vacuum, trions in the charge $q=1$ sector are expected to be more strongly confined to the surface. For a collinear geometry with the electron in between the two holes in analogy to the $\mathrm{H}_{2}^{+}$molecule (see below), the trionic wavefunctions should be localized in the surface. (We do not include in these calculations additional orbitals on virtual sites above the surface.) Consequently, our MCSCF result giving an excitation energy of $E_{\text {exc }}=10.8 \mathrm{eV}$ for a large cluster $\left(\mathrm{F}_{13} \mathrm{Li}_{37}\right)$ in the $q=0$ sector should be compared with the BSE result for surface-confined excitons. We obtain satisfactory agreement on the $10 \%$ level. We note parenthetically that such a basis set would fail to represent excitations in the $q=-1$ sector
TABLE I. Comparison to experimental estimates (Ref. 11) for minimum $\left(\min E_{\text {trion }}\right)$ and maximum trion excitation energies ( $\max E_{\text {trion }}$ ), ionization energy of the charged cluster (second $E_{\text {ioniz }}$ ), and binding energy $\left(E_{\text {bind }}\right)$ defined as the difference between $\max E_{\text {trion }}$ and second $E_{\text {ioniz. }}$. The error interval includes calculation results from smaller clusters.

\begin{tabular}{lccc}
\hline \hline & Experiment & $\mathrm{F}_{13} \mathrm{Li}_{37}$ & Error interval \\
\hline $\min E_{\text {trion }}[\mathrm{eV}]$ & 12.6 & 10.5 & $10.1-11.6$ \\
$\max E_{\text {trion }}[\mathrm{eV}]$ & & 12.1 & $11.7-13.0$ \\
$\operatorname{second} E_{\text {ioniz }}[\mathrm{eV}]$ & & 14.8 & $14.3-16.9$ \\
$E_{\text {bind }}[\mathrm{eV}]$ & $3.5 \pm 1$ & 2.2 & $1.6-3.5$ \\
\hline \hline
\end{tabular}

(Fig. 5) such as image states weakly bound and delocalized above the surface.

The excitation energy of trions is defined as the totalenergy difference between the ground state and the excited state of the singly charged cluster. Since in ion-surface scattering experiments the excitation is "broad band," i.e., not spectrally selective, the "first" hole can be generated anywhere in the valence band. Accordingly, the minimum excitation energy is given by the energy difference of the trion state and the state with the initial hole at the bottom of the valence band (see Fig. 4). The maximum excitation energy corresponds to the hole at the top of the valence band or, equivalently, to the ground state of the $q=1$ ionic system. Results for excitation energies and binding energies are displayed in Table I. The maximum excitation energy obtained from the MCSCF calculations is $12.1 \mathrm{eV}$ for the largest cluster. Results for smaller clusters give an error interval from 11.7 to $13 \mathrm{eV}$. The excitation energy derived from experiment ${ }^{11}$ was $12.6 \mathrm{eV}$, in good agreement with the present results. The size of the nonmonotonic fluctuations of the trion excitation energies with increasing cluster size (Fig. 6) is within the accuracy level we aim for (about $1 \mathrm{eV}$ ).

Among the energies for the different clusters, we find (Fig. 6) slightly but systematically lower values for $\mathrm{F}_{5}$-based clusters with a fluorine in the center $\left[\mathrm{F}_{5} \mathrm{Li}_{17}, \mathrm{~F}_{9} \mathrm{Li}_{25}\right.$ (for both one and two layers, see Fig. 2), and $\left.\mathrm{F}_{13} \mathrm{Li}_{37}\right]$ compared to $\mathrm{F}_{4}$-based clusters with a lithium in the center $\left(\mathrm{F}_{4} \mathrm{Li}_{13}, \mathrm{~F}_{5} \mathrm{Li}_{14}\right.$, and $\mathrm{F}_{12} \mathrm{Li}_{33}$ ). This can be understood from the symmetry of the system. For the trion state, two holes have to be distributed over nearby fluorine sites. The configuration of one hole in the center and the wavefunction of the second hole symmetrically distributed over surrounding fluorine sites is a configuration of higher symmetry in the finite cluster and thus energetically more favorable than a distribution where none of the holes is located in the center of symmetry. This leads to slightly higher trion energies in $\mathrm{F}_{4}$-based clusters than in $\mathrm{F}_{5}$-based clusters.

As we are interested in the energetically lowest trion state, the largest cluster used in our study is chosen such that the central lattice site is occupied by a fluorine. In Fig. 6 the results for two $\mathrm{F}_{9}$ clusters are shown. Although they have the same number of active fluorine and lithium ions, the trion energies obtained are lower for the $\mathrm{F}_{9}$ cluster with all fluorine ions in the surface layer compared to the results for the $\mathrm{F}_{9}$ cluster with the fluorine ions distributed over two layers 


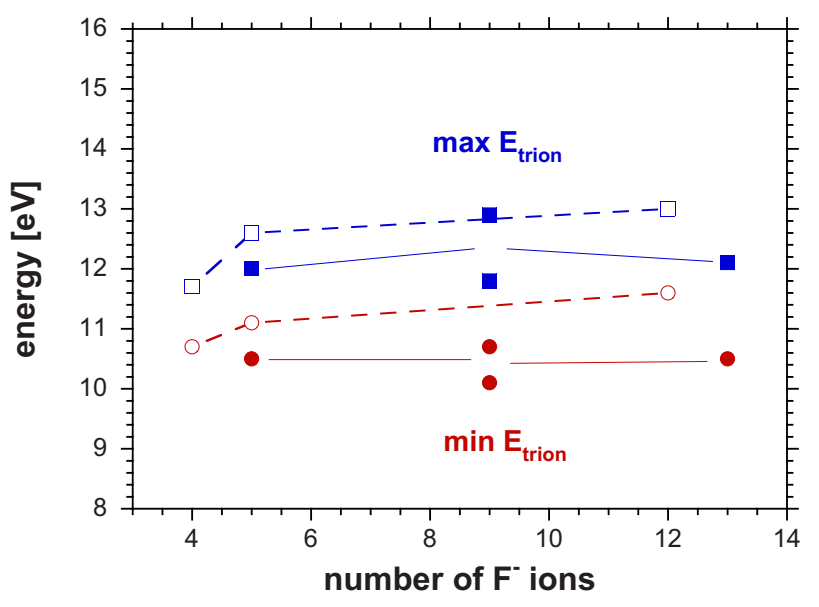

FIG. 6. (Color online) Minimum (min $E_{\text {trion }}$, red dots) and maximum trion excitation energy ( $\max E_{\text {trion }}$, blue squares) as a function of cluster size represented by the number of fluorine ions (lines to guide the eyes). To display the systematic difference between $\mathrm{F}_{5}$-based clusters $\left[\mathrm{F}_{5} \mathrm{Li}_{17}, \mathrm{~F}_{9} \mathrm{Li}_{25}\right.$ (for both one and two layers, see Fig. 2), and $\left.\mathrm{F}_{13} \mathrm{Li}_{37}\right]$ and $\mathrm{F}_{4}$-based clusters $\left(\mathrm{F}_{4} \mathrm{Li}_{13}, \mathrm{~F}_{5} \mathrm{Li}_{14}\right.$, and $\mathrm{F}_{12} \mathrm{Li}_{33}$ ) we distinguish between $\mathrm{F}_{5}$-based (full symbols) and $\mathrm{F}_{4}$-based clusters (open symbols). Both the single and doublelayered $\mathrm{F}_{9}$ clusters are $\mathrm{F}_{5}$ based. The $\mathrm{F}_{9}$ data points with lower energy values correspond to the $\mathrm{F}_{9}$ cluster with all $\mathrm{F}$ in the surface layer while the higher values are for the two-layered $\mathrm{F}_{9}$ cluster, see text.

(see Fig. 2). The reason for this energy difference will become clear in the next section. We will show that the two holes of the trion tend to occupy next-to-nearest-neighbor $\mathrm{F}^{-}$ sites which is suppressed in the two-layer cluster as there are only five fluorine ions in the topmost layer. Consequently, the energetically most favorable configuration with one hole in the center of the cluster and the second hole at a next-tonearest-neighbor $\mathrm{F}^{-}$site is only possible in the single-layer $\mathrm{F}_{9}$ cluster.

The trion binding energy is given by the energy difference to the ground state of the doubly charged $(q=2$, two-hole) system. Unlike for the excitation energy, a correction for a finite-size cluster calculation is more important for the binding energy and should be included. For the $q=2$ ground state the three charges should have reached asymptotic distances. Within our calculation, only the electron can be regarded as infinitely distant in this case. Due to the finite cluster size, possible values for the distance $R_{h h}$ between the residual two holes lie between 5.4 and 21.5 a.u. (in the $\mathrm{F}_{13} \mathrm{Li}_{37}$ cluster) where the spatial distribution of the MOs occupied by the holes indicate a largest possible separation of the holes, i.e., $R_{h h}=21.5$ a.u. for the $\mathrm{F}_{13} \mathrm{Li}_{37}$ cluster. We therefore subtract the screened hole-hole interaction, $\left[\epsilon(\omega) R_{h h}\right]^{-1}$, from the trion energy, where $\epsilon(\omega)$ is the dielectric constant. We note that $\epsilon(\omega)$ determines the long-range electronic screening in the crystal. The use of $\left[\epsilon(\omega) R_{h h}\right]^{-1}$ for the hole-hole interaction is a first-order approximation. However, as the hole-hole distance considered spans several lattice constants this approximation should give a value for the hole-hole interaction within the accuracy level of the present study. As a first-order estimate, the optical value for $\mathrm{LiF}, \epsilon(\omega)=1.96,{ }^{32}$ can be used. For an improved estimate we take into account the depen- dence of $\epsilon(\omega)$ on the ratio of the hole-hole distance to the mean hole velocity $\left\langle v_{h}\right\rangle$ (Ref. 33) with $\left\langle v_{h}\right\rangle=0.22$ a.u. being the mean velocity in $\mathrm{LiF}$. The ratio $R_{h h} /\left\langle v_{h}\right\rangle \approx 98$ in the $\mathrm{F}_{13} \mathrm{Li}_{37}$ corresponds to an $\epsilon(\omega) \approx 2.7$ according to the dielectric response function calculated by Hägg et al. ${ }^{33}$ We note parenthetically that in the latter case, the response function was determined for the perturbation by an external charge above the surface. However, as the induced potential for charges very close to the surface should be similar, ${ }^{34}$ we expect the obtained value for $\epsilon(\omega)$ to be an acceptable approximation. We therefore use $\epsilon(\omega) \approx 2.7$ to calculate the trion binding energy (Table I). We determine an "error bar" of the excitation and binding energies (Table I) from the (nonmonotonic) fluctuations of the results with cluster size where for every cluster the size-dependent dielectric screening is used.

To determine the binding energy of a trion, the groundstate energy of the doubly charged cluster was calculated to obtain the second ionization energy of the LiF cluster (see Fig. 4). The latter may be affected by the uncertainty in the ground-state determination: As we use state averaged MC$\mathrm{SCF}$, the average total energy of all states is minimized during the calculation. As a consequence, the ground-state energy of the singly charged cluster obtained in our calculations is slightly higher than the result would be in the case of a one-state calculation. To be consistent, all possible nearly degenerate "ground states" of the doubly charged cluster should be calculated. If there are $n \mathrm{~F} 2 p$-like MOs considered in the calculation, there are $n$ different possibilities for the location of one hole (in the singly charged cluster) while for two holes (in the doubly charged cluster) already $n^{2}$ different configurations are possible in the ground state. As a consequence, the simultaneous optimization of 16 states in the case of $\mathrm{F}_{4} \mathrm{Li}_{13}$ and of 121 states in the case of $\mathrm{F}_{13} \mathrm{Li}_{37}$ would be necessary, which goes beyond our present capabilities. We therefore calculate only the lowest, "real" ground state of the doubly charged cluster. To get an estimate for the shift of the ground-state energy when all ground states are considered in the MCSCF calculation, we have studied the effect on the doubly charged $\mathrm{F}_{4} \mathrm{Li}_{13}$ cluster where, for computational reasons, we restricted the number of active states to 14 . The energy difference compared to the one-state calculation was below $0.3 \mathrm{eV}$.

For all clusters, the second ionization energy determined from MCSCF calculations exceeds the trion excitation energy so that the trion state is, indeed, a bound state. We obtain a trion binding energy $E_{\text {bind }}$ of about $2.2 \mathrm{eV}$ with an error interval due to finite cluster size corrections ranging from 1.6 to $3.5 \mathrm{eV}$. This is consistent with the estimated energy of $3.5 \pm 1 \mathrm{eV}$ found in experiment. ${ }^{11,12}$

\section{B. Trion wavefunction}

We present now results for the "wavefunction" of the trion, more precisely, for the pair correlation function for its two holes. We evaluate the joint probability distribution function $\rho\left(\vec{r}_{1}^{(h)}, \vec{r}_{2}^{(h)}\right)$ of the two holes. Fixing the first hole at the central F site, $\rho\left(0, \vec{r}_{2}^{(h)}\right)$ gives the pair correlation function or conditional probability for finding the second hole of the 


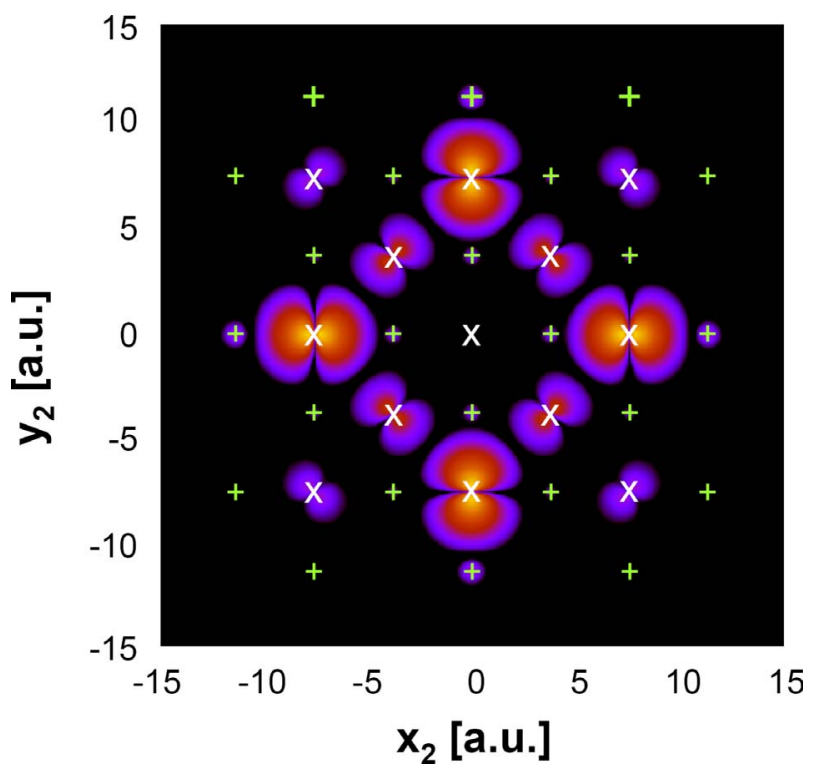

FIG. 7. (Color online) Pair correlation function (conditional probability distribution) for the second hole at the surface $\left(z_{2}=0\right)$ when the first hole is fixed at $x_{1}=y_{1}=z_{1}=0$ for an $\mathrm{F}_{13} \mathrm{Li}_{37}$ cluster. The position of the ions is also marked $\left(\times: \mathrm{F}^{-},+\mathrm{Li}^{+}\right)$.

bound complex at $\vec{r}_{2}$ when one hole resides at the central $\mathrm{F}$ site of the cluster. This quantity is, apart from conceptual aspects, also of interest for the collisional interaction process: the excitation of a trion during the impact of positive ions on an LiF surface can only be an efficient charge exchange and energy-loss channel when the trionic state is well localized so that a sufficient overlap of the corresponding wavefunctions can occur during the short interaction time window.

To determine the pair correlation function we calculate the two-hole wavefunction $\Psi^{(h)}\left(\overrightarrow{\mathbf{r}}_{1}, \overrightarrow{\mathbf{r}}_{2}\right)$ which is given, after tracing out of all other degrees of freedom, by the superposition of different two-hole Slater determinants,

$$
\Psi^{(h)}=\sum_{l} \alpha_{l} \Phi_{l}^{(h)},
$$

where $\Psi^{(h)}$ depends on $\overrightarrow{\mathbf{r}}_{1}$ and $\overrightarrow{\mathbf{r}}_{2}$, which include all spacial and spin coordinates of the holes. The difference to Eq. (1) is that the Slater determinants $\Phi_{l}^{(h)}$ include only the two molecular orbitals $\phi_{j_{1}, l}$ and $\phi_{j_{2}, l}$ of the holes,

$$
\Phi_{l}^{(h)}=\frac{1}{\sqrt{2}}\left|\begin{array}{ll}
\phi_{j_{1}, l}\left(\overrightarrow{\mathbf{r}}_{1}\right) & \phi_{j_{1}, l}\left(\overrightarrow{\mathbf{r}}_{2}\right) \\
\phi_{j_{2}, l}\left(\overrightarrow{\mathbf{r}}_{1}\right) & \phi_{j_{2}, l}\left(\overrightarrow{\mathbf{r}}_{2}\right)
\end{array}\right| .
$$

For a given spin orientation of the holes, $\left|\Psi^{(h)}\left(\vec{r}_{1}, \vec{r}_{2}\right)\right|^{2}$ gives, up to a normalization constant, the probability for finding one hole at the point $\vec{r}_{1}$ and the other hole at $\vec{r}_{2}$. A typical result for the pair distribution function $\rho\left(0, \vec{r}_{2}\right)=\mid \Psi^{(h)}\left(\vec{r}_{1}\right.$ $\left.=0, \vec{r}_{2}\right)\left.\right|^{2}$ (the first hole occupies the central fluorine site) in the surface plane $\left(z_{2}=0\right)$ is shown in Fig. 7 for an $F_{13}$ cluster. The probability density has the symmetry expected for the lowest excited state. It shows very pronounced maxima at the next-to-nearest-neighbor fluorine sites, while the probability to find the second hole at a nearest-neighbor $\mathrm{F}$ is

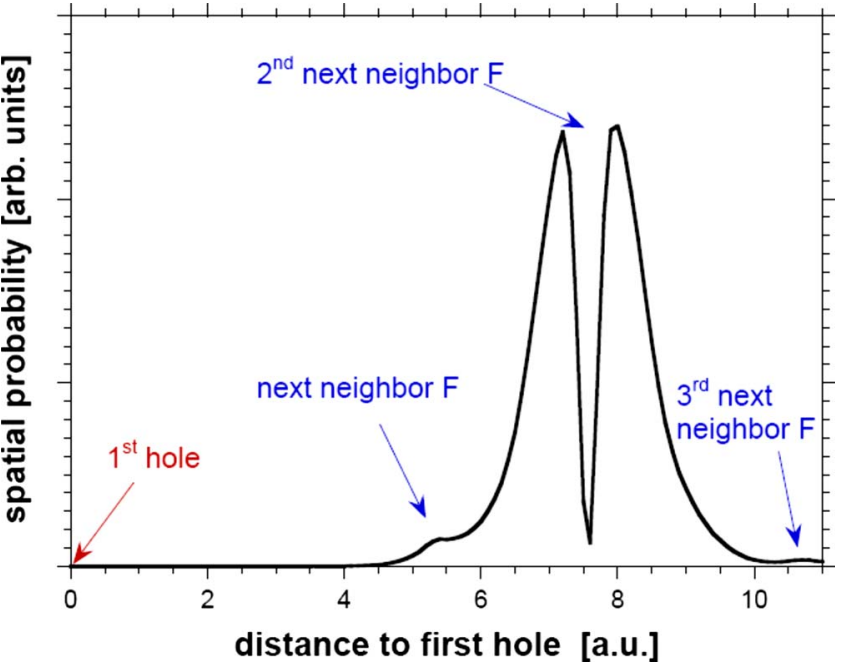

FIG. 8. (Color online) Radial probability distribution $\mid \Psi^{(h)}\left(\mid \vec{r}_{1}\right.$ $\left.-\vec{r}_{2} \mid\right)\left.\right|^{2}$ where $\vec{r}_{1}=0$ and $z_{2}=0$.

smaller by an order of magnitude and even smaller at a thirdnearest-neighbor $\mathrm{F}$ site. This can be understood in view of the position of the excited electron which is located at the $\mathrm{Li}^{+}$ site between the two holes because of the Coulomb attraction of the lithium ion. It is therefore energetically favorable for the holes to be located at two sites adjacent to the electron which are next-to-nearest $\mathrm{F}$ sites. In other words, the electron-bihole complex forms a collinear three-center "quasimolecule" as (schematically) illustrated in Fig. 1(b). Note the $p$-orbital character of the four main peaks with a clearly visible nodal plane through the exact position of the fluorine lattice sites. As expected, we find a correlation hole, i.e., vanishing probability to find the second hole at the exact location of the first hole $(x=y=z=0)$.

The "radial" probability distribution $\left|\Psi^{(h)}\left(\left|\vec{r}_{1}-\vec{r}_{2}\right|\right)\right|^{2}$ corresponding to Fig. 7 is plotted in Fig. 8. The expectation value for $\left|\vec{r}_{1}-\vec{r}_{2}\right|$ is 7.62 a.u. coinciding with twice the lattice constant of $\mathrm{LiF}$ (distance between an $\mathrm{F}$ and an adjacent $\mathrm{Li}$ atom) $a=3.8$ a.u. Due to the $p$ character of the hole orbital (see Fig. 7) the distribution shows a double peak at the corresponding distance. The dip within the double peak originates from the nodal planes of the orbitals, however, smeared out somewhat by the spherical average. Our results confirm the conclusion drawn from experiment ${ }^{11}$ that the two holes of a trion are located in close proximity to each other. As opposed to Wannier excitons, the excited trion is well localized. The MO occupied by the excited electron is the energetically lowest of the three $\mathrm{Li} 2 s$-like MOs included in the calculation as expected for the first (lowest) trion state. The dominant weight of the wavefunction lies at the lithium site between the two holes.

In comparing the trion problem (excitation of the charged cluster) with the exciton problem (excitation of the neutral cluster), the question arises whether a trion configuration is possible with the excited electron protruding into vacuum as is the case for surface excitons. There, the inclusion of basis sets in the vacuum allowing the electron wavefunction to be centered above the surface leads to a $25 \%$ reduction in the excitation energy. ${ }^{17,18}$ Due to the stronger attraction of the 
electron to the two holes of the trion, we expect electron states in the vacuum to be of less importance for trion energies. For computational reasons, basis functions centered outside the cluster surface have therefore not been included in most of our calculations. However, in order to clarify if such a configuration may be energetically more favorable than the surface confined trion, we have performed test calculations on the $\mathrm{F}_{9} \mathrm{Li}_{25}$ cluster. In order to make the calculation computationally feasible a smaller basis for the fluorine ions was used with two $s$ and one $p$ Gaussians. The set of basis functions was extended by three $s$ and one $p$ Gaussians including a diffuse $s$ orbital for every virtual lattice site in the vacuum, centered above the five central fluorine ions as well as the four central lithium ions at $z=1.9$ and 3.8 a.u. The use of a larger basis set or a larger cluster would go beyond the present abilities of the code. We note that the present test calculations are not converged with respect to basis set or cluster size. MCSCF calculations on the charged cluster yield, in fact, a trion configuration where the electron wavefunction is dominated by MOs built up by the vacuum basis functions with additional contributions from basis functions of the central lithium ions in the surface. The excitation energy as well as the binding energy of this trion are, however, comparable to the collinear case within the accuracy level of the method. We obtain a binding energy of $E_{\text {bind }} \simeq 2.3 \mathrm{eV}$ for the calculation without basis functions in the vacuum and $E_{\text {bind }} \simeq 2.7 \mathrm{eV}$ when additional basis functions are used. This $17 \%$ increase in binding energy is rather small compared to the increase in binding energy of excitons (i.e., in the neutral system) when vacuum basis functions are included in the calculation. The exciton binding energy obtained from calculations including basis sets in the vacuum is more than twice the binding energy for the surface confined exciton. ${ }^{17,18}$ From our test calculations we conclude that a trion configuration with the electron located above the surface is possible but does not necessarily correspond to the energetically lowest trion state. Of course, such trion configurations should be included in dynamic calculations of trion excitation.

Of particular interest is the spatial distribution of the trion configuration with the electron wavefunction protruding into vacuum. As opposed to the typical collinear alignment of a three-body Coulomb complex, the three charges form a triangle in the (100) plane normal to the (001) surface plane. As our test calculations including basis functions in the vacuum are not fully converged in cluster size (using more than $9 \mathrm{~F}^{-}$is computationally not feasible) it is difficult to draw definite conclusions about the localization of the two holes.

In the present work, we exclusively study surface trions. In principle, the method employed could also be used to study trions in the bulk. However, the number of active ions needed to simulate a bulk cluster is considerably larger. If we assume, as in the case of surface trions, a next-to-nearestneighbor configuration of the two holes, a bulk cluster with at least $47 \mathrm{~F}^{-}$and $100 \mathrm{Li}^{+}$ions is needed to study trions in the bulk. This clearly exceeds the present computational capabilities. Based on the present work on surface trions we expect that bulk trions are bound states as well. The trion excitation and binding energies in the bulk are expected to slightly differ from the corresponding values at the surface

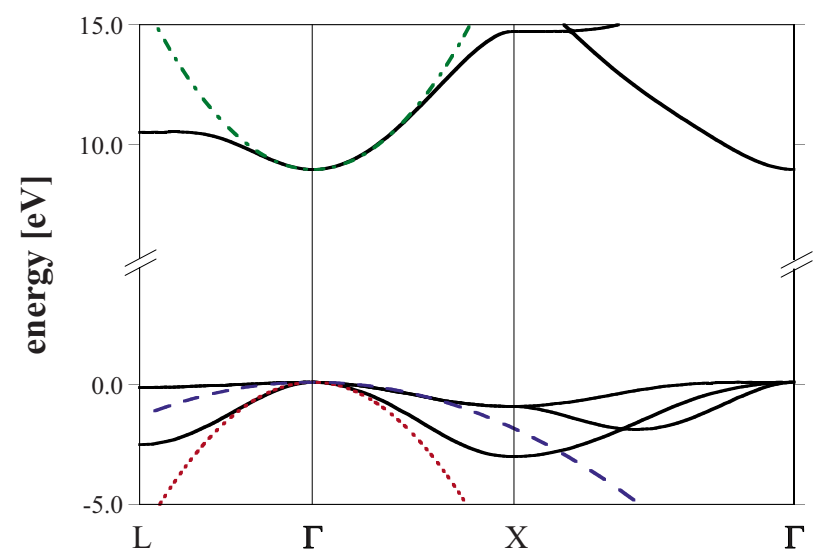

FIG. 9. (Color online) Band structure of LiF. Shown are the valence bands and the lowest conduction bands (solid lines). Fits to parabolas near the $\Gamma$ point (dashed, dotted, and dashed-dotted lines) give the effective masses (see text).

due to the different screening by the crystal environment. The spatial distribution of a bulk trion will most likely also be a collinear configuration. To verify this hypothesis quantitatively a full $a b$ initio calculation on a bulk cluster is necessary.

\section{RELATION TO THE THREE-BODY COULOMB PROBLEM}

The trion is a bound state of the three-body Coulomb system embedded in the solid environment. It is now instructive to compare this state with other Coulomb three-body systems in vacuum as well as states in confined space of lower dimension.

Key input parameters for such a comparison are the effective masses of the holes, $m_{h}^{*}$, and the electron, $m_{e}^{*}$, which represent, to first order, the coupling of the quasiparticles to the periodic potential of the solid. The effective masses were determined from band-structure calculations of LiF (Ref. 35) using the relation $1 / m^{*}=\partial^{2} E(k) / \partial k^{2}$ (in atomic units) where $E(k)$ is the band energy as a function of the wave vector $k$. The three valence bands and the lowest conduction bands of LiF are shown in Fig. 9 together with fits to parabolas near the $\Gamma$ point to determine the curvature of $E(k)$. As there are three valence bands, two of which are degenerate at the $\Gamma$ point, a mean value for the effective mass of the hole was calculated via $\frac{1}{m_{h}^{*}}=\frac{1}{3}\left(\frac{1}{m_{1}^{*}}+\frac{2}{m_{2}^{*}}\right)$, where $m_{2}^{*}$ and $m_{1}^{*}$ are the effective masses in the degenerate and in the nondegenerate valence bands, respectively. We find the effective masses for electrons and holes to be $m_{e}^{*}=0.948 m_{e}$ and $m_{h}^{*}=2.227 m_{e}$.

The two holes are the "heavy" particles and the electron is the "light" particle. This suggests an analogy to the oneelectron molecular ion, e.g., $\mathrm{H}_{2}^{+}$. The binding energy defined as the difference between the minimum energy at the equilibrium distance of the nuclei $\left(R_{\min }=2.00\right.$ a.u. $)$ and the asymptotic energy of $\mathrm{H}_{2}^{+}$amounts to $2.79 \mathrm{eV}$. The $\mathrm{H}_{2}^{+}$ion corresponds to a limiting case of three interacting charges where two of them can be regarded as infinitely heavy compared to the third light particle so that the reduced mass of the system $\mu \approx 1$ in units of the light mass. This is clearly 


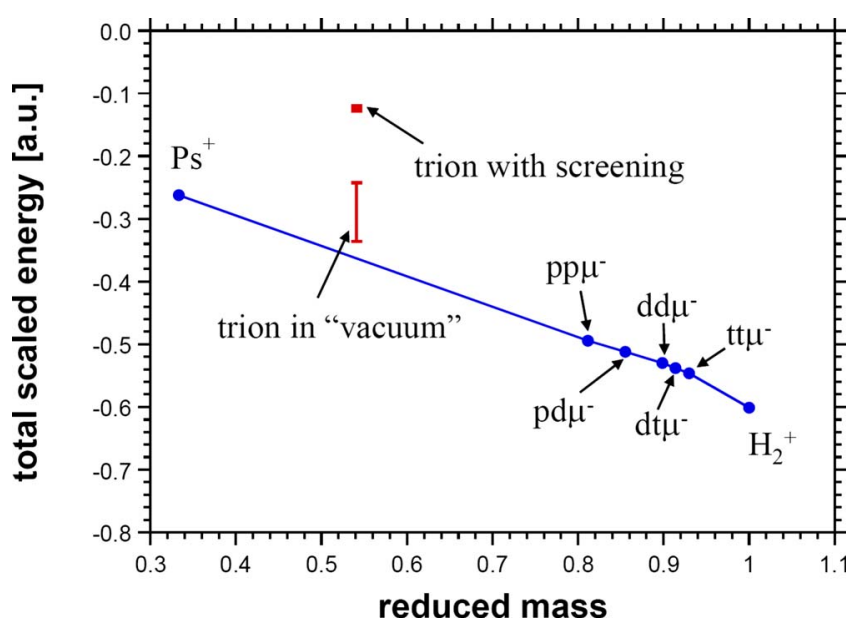

FIG. 10. (Color online) Total scaled energy of the three-body systems $\mathrm{H}_{2}^{+}, \mathrm{Ps}^{-}, p p \mu^{-}$(Ref. 36), $p d \mu^{-}, d d \mu^{-}, d t \mu^{-}, t t \mu^{-}$(Ref. 37), and a trion as a function of the reduced mass of the system in units of the light mass.

very different from the system of two holes with $m_{h}^{*}$ $=2.227 m_{e}$ binding an electron with $m_{e}^{*}=0.948 m_{e}$. The corresponding reduced mass of $\mu=0.54$ (in units of $m_{e}^{*}$ ) is much closer to that of the opposite limit of three particles with equal mass which is realized by the charged positronium ion $\operatorname{Ps}^{-}\left(e^{-} e^{+} e^{-}\right)$with $\mu=1 / 3$. Other three-body systems in between these limiting cases are muonic molecules such as, for example, $p p \mu^{-}$with a reduced mass of $\mu=0.812$ (in units of $\left.m_{\mu}\right)$. The total energy of these three-body systems scaled by the factor $1 / m_{1}$, where $m_{1}$ is the mass of the light particle, shows an approximately linear dependence on the reduced mass of the system (Fig. 10). It is now instructive to relate our present result for trions to this "universal" bindingenergy scaling. To do so, we have to take into consideration corrections due to the solid state environment. To first order, this three-body Coulomb problem is embedded in a dielectric medium with a dielectric constant $\epsilon(\omega)$. The Coulomb interaction between two charges $Q_{1}$ and $Q_{2}$ will be screened by a factor $1 / \epsilon$,

$$
\frac{Q_{1} Q_{2}}{r_{1,2}} \rightarrow \frac{Q_{1} Q_{2}}{\epsilon r_{1,2}},
$$

where $r_{1,2}$ is the distance between the two charges. The effect of screening manifests itself in a shift of the energy curve toward lower (absolute) energies and a reduced binding energy. For a comparison with the scaled binding energy of the three-body Coulomb problem in vacuum, we have to remove the screening by multiplying the scaled trion energy by $\epsilon$. This corresponds to a trion in vacuum, i.e., a free three-body system of $\mu=0.54$. The resulting energy is now close to the linear curve of the free three-body systems. The limits of the error bar in Fig. 10 correspond to the use of the optical limit $\epsilon(\omega)=1.96$ for the dielectric constant (upper energy limit) and the use of the value $\epsilon(\omega) \approx 2.7$ estimated in Sec. III A (lower limit).
Within the framework of a three-body Coulomb problem in a screening environment, Thilagam ${ }^{13}$ derived an analytical expression for the binding energy of a trion in a twodimensional electron system in order to provide an order of magnitude estimate. His description was based on a threebody Hamiltonian of the two holes and the bound electron where the three charges are assumed to form a line. In this model, the effect of the periodic crystal potential is included only through the effective masses of electrons and holes. The charges are thus treated as free particles (with effective masses) moving in a dielectric medium. Thilagam's expression for the trion binding energy reads

$$
E_{\text {bind }}=\left(\frac{s^{2}+4 s+2}{s^{2}+6 s+3} \cdot \frac{9}{4}-1\right) s \frac{M_{R} R_{H}}{\epsilon^{2} m_{e}} .
$$

Here, $s=m_{h}^{*} / m_{e}^{*}$, with $m_{e}^{*}$ and $m_{h}^{*}$ being the effective masses of the electron and the holes, respectively. $M_{R}=m_{e}^{*} m_{h}^{*} /\left(m_{e}^{*}\right.$ $\left.+m_{h}^{*}\right)$ is the reduced effective mass, $m_{e}$ is the free electron mass, and $R_{\mathrm{H}}$ denotes the Rydberg constant. As the hole-hole distance is not described in Ref. 13, we have here used the optical limit of the dielectric constant $\epsilon=1.96$ for $\mathrm{LiF}$. Remarkably, Eq. (5) predicts a binding energy close to the present results $\left(E_{\text {bind }}=3.8 \mathrm{eV}\right)$. The surprisingly good agreement should be taken with caution given the simplicity of the model, in particular the neglect of the crystal potential.

\section{CONCLUSION}

Employing the embedded cluster approach, we have performed $a b$ initio calculations on the multiconfiguration selfconsistent field level to study trions on an LiF surface. We have shown that their formation is possible. We find a trion excitation energy $E_{\text {exc }}$ in the range of 10.5 to $12.1 \mathrm{eV}$ (with an error interval $10.1-13 \mathrm{eV}$ ) and a binding energy $E_{\text {bind }}$ $=2.2$ (error interval $1.6-3.5 \mathrm{eV}$ ) relative to the ground state of the doubly charged cluster. Trions have larger binding energies than excitons in the neutral system. From the determination of the hole-hole pair correlation function we deduce that the two holes are predominantly located at next-tonearest-neighbor F sites with the electron "in between." The trion in the surface forms a linear (quasi) molecule.

Future extensions of this work should consider trion excitations with non-collinear geometry. Such a configuration may represent an excited state of a trion with the electron protruding into vacuum. Moreover, the dynamical excitation process in charged-particle or photonic interactions with the surface is largely unknown and of considerable interest.

\section{ACKNOWLEDGMENTS}

This work was supported by the FWF Austria under Project No. P17449-N02, by the ÖAD under Project No. FR 02/2007, by the PHC Amadeus of the French Ministry of Foreign and European Affairs, and by EU under Contract No. HPRI-CT-2005-026015. We thank H. Lischka and M. Ruckenbauer for their helpful assistance with COLUMBUS. 
${ }^{1}$ M. A. Lampert, Phys. Rev. Lett. 1, 450 (1958).

${ }^{2}$ B. Stébé and G. Munschy, Solid State Commun. 17, 1051 (1975).

${ }^{3}$ B. Stébé and C. Comte, Phys. Rev. B 15, 3967 (1977).

${ }^{4}$ B. Stébé, T. Sauder, M. Certier, and C. Comte, Solid State Commun. 26, 637 (1978).

${ }^{5}$ B. Stébé, E. Feddi, and G. Munschy, Phys. Rev. B 35, 4331 (1987).

${ }^{6}$ R. Schilling and D. C. Mattis, Phys. Rev. Lett. 49, 808 (1982).

${ }^{7}$ R. Schilling and D. C. Mattis, Phys. Rev. B 27, 3318 (1983).

${ }^{8}$ G. A. Thomas and T. M. Rice, Solid State Commun. 23, 359 (1977).

${ }^{9}$ T. Ishii, M. Taniguchi, A. Kakizaki, K. Naito, H. Sugawara, and I. Nagakura, Phys. Rev. B 33, 5664 (1986).

${ }^{10}$ D. Labrie, V. A. Karasyuk, M. K. Nissen, Ya. E. Pokrovskii, and M. L. W. Thewalt, Phys. Rev. B 47, 1628 (1993).

${ }^{11}$ H. Khemliche, J. Villette, A. G. Borisov, A. Momeni, and P. Roncin, Phys. Rev. Lett. 86, 5699 (2001).

${ }^{12} \mathrm{P}$. Roncin, A. G. Borisov, H. Khemliche, A. Momeni, A. Mertens, and H. Winter, Phys. Rev. Lett. 89, 043201 (2002).

${ }^{13}$ A. Thilagam, Phys. Rev. B 55, 7804 (1997).

${ }^{14}$ M. Shibata, Y. Kuramoto, and T. Watanabe, Phys. Rev. B 42, 2432 (1990).

${ }^{15}$ For a recent review see, e.g., G. Onida, L. Reining, and A. Rubio, Rev. Mod. Phys. 74, 601 (2002).

${ }^{16}$ N.-P. Wang, M. Rohlfing, P. Krüger, and J. Pollmann, Phys. Rev. B 67, 115111 (2003).

${ }^{17}$ M. Rohlfing, N.-P. Wang, P. Krüger, and J. Pollmann, Phys. Rev. Lett. 91, 256802 (2003).

${ }^{18}$ M. Rohlfing, N.-P. Wang, P. Krüger, and J. Pollmann, Surf. Sci. 593, 19 (2005).

${ }^{19} \mathrm{R}$. Shepard, in Ab Initio Methods in Quantum Chemistry II, Advances in Chemical Physics Vol. 69 (Wiley, New York, 1987), pp. 63-200.

${ }^{20}$ A. Schäfer, H. Horn, and R. Ahlrichs, J. Chem. Phys. 97, 2571 (1992); A. Schäfer, C. Huber, and R. Ahlrichs, ibid. 100, 5829 (1994).

${ }^{21}$ Most importantly, correlations due to long-range polarization of the environment are missing. This limitation is inherent to the finite cluster approach. However, since our excitation energies of excitons/trions are calculated as total-energy differences be- tween the ground and excited states of the neutral/singly charged cluster, respectively, the main part of this error will cancel out. The error for the calculated binding energies-where we compare with the total energy of the singly/doubly charged cluster, respectively-may be larger.

${ }^{22}$ P. V. Sushko, A. L. Shluger, and C. R. A. Catlow, Surf. Sci. 450, 153 (2000).

${ }^{23}$ L. Wirtz, J. Burgdörfer, M. Dallos, T. Müller, and H. Lischka, Phys. Rev. A 68, 032902 (2003).

${ }^{24}$ K. S. Song and R. T. Williams, Self-Trapped Excitons, 2nd ed. (Springer, Berlin, 1996).

${ }^{25} \mathrm{H}$. Lischka, R. Shepard et al., computer code columbus, Release 5.9, 2006.

${ }^{26}$ P. A. Cox and A. A. Williams, Surf. Sci. 175, L782 (1986).

${ }^{27}$ K. Saiki, W. R. Xu, and A. Koma, Surf. Sci. 287/288, 644 (1993).

${ }^{28}$ G. Roy, G. Singh, and T. E. Gallon, Surf. Sci. 152/153, 1042 (1985).

${ }^{29}$ T. E. Gallon, Surf. Sci. 206, 365 (1988).

${ }^{30}$ P. Roncin, J. Villette, J. P. Atanas, and H. Khemliche, Phys. Rev. Lett. 83, 864 (1999).

${ }^{31}$ P. Wurz, J. Sarnthein, W. Husinsky, G. Betz, P. Nordlander, and Y. Wang, Phys. Rev. B 43, 6729 (1991).

${ }^{32}$ E. D. Palik and W. R. Hunter, in Handbook of Optical Constants, edited by E. D. Palik (Academic, New York, 1985), p. 675.

${ }^{33}$ L. Hägg, C. O. Reinhold, and J. Burgdörfer, Phys. Rev. A 55, 2097 (1997).

${ }^{34}$ F. J. García de Abajo and P. M. Echenique, Phys. Rev. B 46, 2663 (1992).

${ }^{35}$ The band-dispersion of $\mathrm{LiF}$ is calculated within densityfunctional theory in the local density approximation, using the code ABINIT (Ref. 38). We note that many-body corrections will lead to a considerably larger band gap (Ref. 16), which is, however, of no concern for the effective masses.

${ }^{36}$ O. I. Tolstikhin and M. Matsuzawa, Phys. Rev. A 63, 062705 (2001).

${ }^{37}$ B. P. Carter, Phys. Rev. 165, 139 (1968).

${ }^{38}$ X. Gonze, J.-M. Beuken, R. Caracas, F. Detraux, M. Fuchs, G.-M. Rignanese, L. Sindic, M. Verstraete, G. Zerah, F. Jollet, M. Torrent, A. Roy, M. Mikami, Ph. Ghosez, J.-Y. Raty, and D. C. Allan, Comput. Mater. Sci. 25, 478 (2002). 